\title{
DECOUPLING BETWEEN TORSION AND MAGNETIC FIELDS IN BOUNCING COSMOLOGY AND GALACTIC DYNAMO SEEDS
}

\author{
${ }^{1}$ Garcia de Andrade, L.C. and ${ }^{2}$ A. Ferrandez \\ ${ }^{1}$ Departamento de Fisica Teorica-IF-UERJ-Rua Sao Francisco Xavier 524, Rio de Janeiro, RJ, Maracana, Brazil \\ ${ }^{2}$ Departamento de Matematicas-Universidad de Murcia-Campus de Espinardo, 30100, Murcia, Spain
}

Received 2014-02-05; Revised 2014-03-17; Accepted 2014-03-27

\begin{abstract}
Recently Salim et al. (2007), have shown that galactic dynamo seeds can be possibly attainable in bouncing cosmological models with QED Lagrangeans. In this study we generalise their result by include torsion of spacetime in bouncing cosmology. It is shown that by considering a semi-minimal photon-torsion coupling and a Lagrangean of the type $R F^{2}$ it is possible to find a fast decoupling between magnetic and torsion fields in the contracting phases of the universe. Besides torsion field decays as $K \square a^{\frac{2}{3}}$ while the magnetic field grows as $B \sim a^{-5.5}$ thus explaining the fast decoupling between the two fields. It is expected that at some point of the contracting phase the amplification of the magnetic field may give rise to a enough strong magnetic field to seed a galactic dynamo.
\end{abstract}

Keywords: Torsion Theories, Bouncing Cosmology, Astro-Particle Physics

\section{INTRODUCTION}

Enqvist et al. (1992) place limits on neutrino masses from galactic dynamo mechanism. Since neutrino masses are important in extending the standard model of particle physics, it seems worth to investigate the relation between Lorentz Violation (LV) and galactic dynamos in torsion fields for example (Campanelli et al., 2009; Barrow and Tsagas, 2008). Therefore knowledge of the dynamics between torsion and cosmic magnetic fields may reveal if dynamo mechanism is a powerful mechanism to feed the galactic magnetic fields of nano-Gauss observed in nature. In this letter one shows that by using a scalar electrodynamics in the context of Quantum Electrodynamics (QED) (Drummond and Hathrell, 1980) it is possible to show that magnetic field decays when torsion is fast amplified. It is shown that torsion needed to seed galactic dynamo is of the other of 10$18 \mathrm{~cm}^{-1}$ which can be found in nature and is even weaker than the value estimated in the Early Universe.
In previous work (Barrow and Tsagas, 2008) one notice that semi-minimal coupling has been used on a Lagrangean of the type $\frac{1}{4} R_{i j k l} F^{i j} F^{k l},[\mathrm{i}, \mathrm{j}=0,1,2,3]$. This has provided further constraints on torsion up to $10^{-31} \mathrm{GeV}$. Here though semi-minimal coupling is preserved, we shall use, another gravitational sector in the Lagrangean given by the coupling $R F^{i j} F_{i j}$ as used in the study by (Mazzitelli and Spedalieri, 1988). The term $R_{i j k l} F^{i j} F_{k l}$ displays the same symmetries of LV term, where the Riemann-Cartan curvature tensor, including torsion terms plays the role of the Higgs sector constants $k_{i j k l}$. Here we will not address the problem of the LV since the Lagrangean term used does not favor that. In this study, we show that the use of this photon sector coupled semi-minimally with torsion mode, in scales of $10 \mathrm{kpc}$ would require a not very strong torsion field that might exist in nature, so it seems that the only conclusion is that this necessary imply that galactic magnetic fields can be seeded by a such torsion models also in the (Mazzitelli and Spedalieri, 1988) scalar electrodynamics

(C) 2014 Garcia de Andrade, L.C. and A. Ferrandez. This open access article is distributed under a Creative Commons Attribution (CC-BY) 3.0 license which permits unrestricted use, distribution, and reproduction in any medium, provided the original work is properly credited. DOI: 10.3844/pisp.2014.45.48 
is concerned with semi-minimal coupling of course. Some physicists (Kosteleckly, 2004) argued that torsion is very weak to have time to seed magnetic fields but actually here from Mazzitelli and Spedalieri (1988) scalar QED we have shown that the torsion field may grow exponentially in regions of weak primordial magnetic field which is not strong enough to seed galactic dynamos. The study is organised as follows: In section II we review Mazzitelli and Spedalieri (1988) scalar electrodynamics. In section III we apply the semiminimal coupling to their equations to introduce the torsion field which grows in the contracting phase of the universe as in a bouncing model (Kostelecky et al., 2008), presenting a decoupling between torsion to magnetic fields. Section IV contains conclusions and discussions.

\section{FLAT SEMI-MINIMAL TORSION- PHOTON COUPLING OF $\boldsymbol{R F}^{2}$ LAGRANGEAN}

Though torsion effects are highly suppressed in comparison with curvature ones of Einstein gravity sector, we do not consider here Minkowski space since as can be easily shown here from the field equations that torsion vanishes in Minkowski space Mazzitelli and Spedalieri (1988) Lagrangean is Equation 1:

$$
S=\frac{1}{m^{2}} \int d^{4} x(-g)^{\frac{1}{2}}\left(-\frac{1}{4} F^{2}+\left(m^{2}+\varepsilon R\right) \phi \bar{\phi}-D_{j} \phi D^{j} \bar{\phi}\right)
$$

where, $D_{i}=\partial_{i}-i e A_{i}$ is the covariant derivative for the scalar fields. Mazzitelli and Spedalieri (1988) have computed an effective Lagrangean for the e.m field by integrating the quantum scalar field. Via dimensional regularisation they obtain the effective Lagrangean (Kostelecky et al., 2008) Equation 2:

$$
L_{e f f}=-\frac{1}{4} F^{2}+-\frac{1}{2} \frac{1}{4 \pi_{2}^{d}}\left(\frac{m}{u}\right)^{d-4} \sum a_{j}(x) m^{4-2 j} \Gamma\left(j-\frac{d}{2}\right)
$$

The first Schwinger-De Witt (SDW) coefficients Mazzitelli and Spedalieri (1988) work Equation 3 to 6:

$$
\begin{aligned}
& a_{0}=1 \\
& a_{1}=-\left(\varepsilon-\frac{1}{6}\right) R
\end{aligned}
$$

$$
\begin{aligned}
& a_{2}=\frac{1}{180}\left(R_{i j k l} R^{i j k l}-R_{i j} R^{i j}\right)+\frac{1}{2}\left(\varepsilon-\frac{1}{6}\right)^{2} R^{2}+ \\
& \frac{1}{6}\left(\varepsilon-\frac{1}{5}\right) * R \frac{e^{2}}{12} F^{2} \\
& a_{3}=\ldots+\frac{e^{2}}{60} R_{i j k l} F^{i j} F^{k l}-\frac{e^{2}}{90} R_{i j} F^{i k} F^{k l}+\left(\frac{1}{6}-\varepsilon\right) R F^{2}+\ldots
\end{aligned}
$$

where, we have omitted the Maxwell terms that will not be of our interest in the sequence of the study. Here we note that due to the use of semi-minimal coupling where torsion, which is our only gravitational field, appears only in $\alpha_{2}$ as first term, since in the semi-minimal coupling torsion does not appears in the covariant derivative and consequently not in the electromagnetic field. Actually following this reasoning torsion appears only in the curvatures appears for the first time in $\alpha_{2}$. Following them I shall consider the following effective Lagrangean in Riemann-Cartan spacetime, through the minimal coupling as Equation 7:

$$
L_{e f f}=-\frac{1}{4} F^{2}\left(1+\frac{b}{m^{2}} R\right)
$$

where, we have taken $\mathrm{n}=1$ such as in (Turner and Widrow, 1988; Salim et al., 2007). From this effective Lagrangean we obtain the field equations for the Friedmann spatially flat metric Equation 8 and 9:

$$
d s^{2}=a^{2}\left(-d \eta^{2} d x^{2}\right)
$$

As:

$\partial^{i}\left(F_{i j}\left(1+\frac{b R}{m^{2}}\right)=0\right.$

From these equations one may obtain with appropriated approximations Equation 10:

$$
\left[\ddot{A}_{k}+k^{2} A_{k}\right]\left(1+\frac{b R}{m^{2}}\right)+\frac{b \dot{R}}{m^{2} R} \dot{A}_{k}=0
$$

These equations one may yet approximate for high coherence scales where $\mathrm{k}^{2}<<1$. We also addopt here the fact that in Riemannian case in inflationary epoch $\mathrm{R} \gg>$ $\mathrm{m}^{2}$ so this would reduce the last equation to: 


$$
\left[\ddot{A}_{k}+\frac{\dot{R}}{R} \dot{A}_{k}\right]=0
$$

where, $\mathrm{R}$ is the Ricci scalar. This shows that although there is no inflation here we consider that torsion has a similar behaviour so actually $\dot{K} \gg>m^{2}$.

\section{GALACTIC DYNAMO SEEDS IN RF2 SEMI-MINIMAL COUPLING}

In this section we shall solve Equation 11 in the case of curved spacetime with and performing the semiminimal coupling where the Ricci scalar is approximated taken as $2 \dot{K}$, where $K$ is the time component $K^{0}$, which to simplify matters is the only homogeneous component of contortion, an algebraic combination of torsion. Here we addopt linearisation of the RicciCartan scalar (Kostelecky et al., 2008) Equation 12:

$$
R=g_{i j} R^{i j}=R^{*}+2 \nabla_{i} K^{i}-K^{2}
$$

where, $K^{j}=K_{r}^{r j}$, represents the trace of torsion tensor, $R^{*}$ is the Riemannian Ricci scalar that here shall be taken as constant like in de Sitter or Einstein space, to simplify computations. Minkowski space where it vanishes can be also addressed. Let us now perform the variation of the Lagrangean density $\sqrt{g} L$ with respect to the scale cosmological factor a and contortion K, to complete the system of EinsteinCartan-Maxwell equations of course with propagating torsion. This can be done easily by computing the Euler Lagrange Equation 13 and 14:

$$
\begin{aligned}
& \frac{d}{d t} \frac{\partial \sqrt{g L}}{\partial \dot{a}}-\frac{\partial \sqrt{g L}}{\partial a}=0 \\
& \frac{d}{d t} \frac{\partial \sqrt{g L}}{\partial \dot{K}}-\frac{\partial \sqrt{g L}}{\partial K}=0
\end{aligned}
$$

Let us start from the last equation to determine $\mathrm{K}$ in terms of the scale factor a. This yields Equation 15:

$$
K=-\frac{3 \dot{a}}{a}
$$

Before applying this result to the expression for the Ricci-Cartan scalar, let us express this scalar in terms of the scalar a and torsion trace $\mathrm{K}$. This yields the following expression Equation 16 and 17:

$$
R=g_{i j} R^{i j}=R^{*}+2 \dot{K}^{i}-K^{2}+\partial_{t} \ln \sqrt{g} K
$$

Or:

$R=g_{i j} R^{i j}=-6\left[\frac{\ddot{a}}{a}+\left(\frac{\dot{a}}{a}\right)^{2}\right]+2 \dot{K}^{i}-K^{2}+\left(\partial_{t} \ln a^{3}\right) K$

Which yields Equation 18:

$\dot{R}=\dot{R}^{*}+\ddot{K}+\left(\frac{\dot{a}}{a}+2 K\right) \dot{K}+3\left[\frac{\ddot{a}}{a}-\frac{\dot{a}^{2}}{a^{2}}\right] K$

The expression for $\ddot{K}$ is Equation 19:

$\ddot{K}=-3\left[\frac{\dddot{a}}{a}-3 \frac{\ddot{a}^{3}}{a^{2}}+\left(\frac{\dot{a}}{a}\right)^{3}\right]$

The expression for Ricci-Cartan scalar Lagrangean $\sqrt{g} R$ is Equation 20:

$a^{3} R=-3\left[3 \ddot{a} a^{2}+7 \ddot{a}^{2} a\right]$

Substitution of this expression into the EulerLagrange equation above one has Equation 21:

$\ddot{a} a-4 \ddot{a} \dot{a}=0$

By making use of the ansatz $a \sim t^{n}$ where $\mathrm{n}$ is a real number, one obtains the following algebraic Equation 22:

$n(n-2)-4 n^{2}=0$

Which yields immeadiatly $n=\frac{2}{4}$ and $a \sim t^{-1}$, which represents a contracting phase of the cosmological model with torsion. Therefore from the above expression for $\mathrm{K}$ one obtains $K \square a^{\frac{2}{3}}$. On the other hand the magnetic field undergoes a dynamo like phase undergoing an amplification according to the law $B \sim a^{-5.5}$. This can be easily seen by computing the ratio $\frac{\dot{R}}{R}$ as Equation 23 :

$\frac{R}{R}=\frac{\left[3 \ddot{K}-{ }_{3}^{2}\left(K^{2}\right) \cdot\right]}{\left[3 \dot{K}-{ }_{3}^{2} K^{2}\right]}$ 
Since the torsion is a very weak field this can be approximated to Equation 24:

$$
\frac{R}{R} \approx \frac{\ddot{K}}{\dot{K}}
$$

Substitution into the Maxwell like equation above and solving it and taking into account the expression Equation 25:

$$
B=i k A
$$

where, $\mathrm{B}$ is the magnetic field and $\mathrm{k}$ is the wave number which is given by the inverse of the coherent scale, one obtains the above value of $\mathrm{B} \sim \mathrm{a}^{-5.5}$. Therefore one may conclude that the torsion decays while the magnetic field grows in the contracting phase of the universe (Salim et al., 2007) exactly like in the general relativistic version investigated by Salim et al. (2007; Novello and Bergliaffa, 2008).

\section{DISCUSSION AND CONCLUSION}

In this study we show that a semi-minimal torsion coupling in Riemann-Cartan spacetime can be used to generalize previous papers on QED cosmology where a Minkowski space with torsion has been used. AsLagrangeans can be used to determine the torsion which can be used to seed galactic dynamos.

The motivation from this study came from some work by Campanelli et al. (2009) where they investigate similar subjects in the general relativistic backgrounds of Riemannian geometry and by the work of Salim et al. (2007) on the amplification of the magnetic field in bouncing cosmological models. As in their case we think some improvements can be made in obtained galactic dynamo seeds if one uses the matter content of the universe instead of the vacuum QED in curved spacetimes with torsion used here. This may appear elsewhere.

\section{ACKNOWLEDGEMENT}

We would like to express our gratitude to $\mathrm{V}$. Lemes, Ariel Zithnitsky, A Maroto and D Sokoloff for helpful discussions on the subject of this study. Special thanks $\mathrm{n}$ anonymous referee for his extremely kind suggestions which allowed us to making considerable and important improvements on the first draft of this letter. One of us (GdA) would like to thank financial supports from CNPq. and University of State of Rio de Janeiro (UERJ).

\section{REFERENCES}

Barrow, J.D. and C. Tsagas, 2008. Publisher's note: Slow decay of magnetic fields in open friedmann universes. Phys. Rev. D, 77: 107302-107302. DOI: 10.1103/PhysRevD.77.109904

Campanelli, L., P. Cea and G.L. Fogli, 2009. Lorentz symmetry violation and galactic magnetism. Phys. Lett., B, 680: 125-128. DOI: 10.1016/j.physletb.2009.08.041

Drummond, I.T. and S.J. Hathrell, 1980. QED vacuum polarization in a background gravitational field and its effect on the velocity of photons. Phys. Rev. D, 22: 343-355. DOI: 10.1103/PhysRevD.22.343

Enqvist, K., P. Olesen and V. Semikoz, 1992. Galactic dynamo and nucleosynthesis limit on the Dirac neutrino masses Phys. Rev. Lett., 69: 2157-2159. DOI: 10.1103/PhysRevLett.69.2157

Kosteleckly, A., 2004. Gravity, Lorentz violation and the standard model. Phys. Rev. D, 69: 105009-105009. DOI: 10.1103/PhysRevD.69.105009

Kostelecky, A., N. Russell and J. Tasson, 2008. Constraints on Torsion from Bounds on Lorentz Violation. Phys. Rev. Lett., 100: 111102-111102. DOI: 10.1103/PhysRevLett.100.111102

Mazzitelli, D.F. and J.P. Spedalieri, 1988. Simple form for the Gaussian equations in curved space. Phys. Rev. D, 37: 3533-3533. DOI: 10.1103/PhysRevD.37.3533

Novello, M. and S.E.P. Bergliaffa, 2008. Bouncing cosmologies. Phys. Reports, 463: 127-213. DOI: 10.1016/j.physrep.2008.04.006

Salim, J.M., N. Souza, S.E.P. Bergliaffa and T. Prokopec, 2007. Creation of cosmological magnetic fields in a bouncing cosmology. J. Cosmol. Astroparticle Phys., 0704: 011-011. DOI: 10.1088/1475-7516/2007/04/011

Turner, M.S. and L.M. Widrow, 1988. Inflationproduced, large-scale magnetic fields. Phys. Rev. D, 37: 2743-2743. DOI: 10.1103/PhysRevD.37.2743 